MARTA KaŹMIERCZAK ORCID: 0000-0003-2925-0209

Université de Varsovie mkazmierczak@uw.edu.pl

\title{
UNE THÉORIE ITINÉRANTE? \\ LA PENSÉE TRADUCTOLOGIQUE OCCIDENTALE DANS LA TRADUCTION RUSSE (RÉCEPTION ÉDITORIALE)
}

\section{OBJECTIFS ET LIMITES DE L’ÉTUDE}

Comme le souligne Ewa Kraskowska, « Ce qui est traduit en des langues différentes, et par qui, voilà ce qui détermine en grande partie [...] l'étendue et la façon dont sont diffusés les concepts spécifiques ou les courants de recherche dans l'univers académique $»^{1}$. En partant du principe que la théorie de la traduction peut être une «théorie itinérante » (travelling theory), je me propose d'examiner ici comment elle voyage de l'ouest vers l'est. Je me concentrerai sur la réception éditoriale de la traductologie occidentale, ou plus précisément, sur le nombre et le choix des ouvrages traduits et publiés en Union soviétique puis en Russie après $1991^{2}$. La «traductologie occidentale » envisagée ici n'est pas à rapprocher de «l'Europe occidentale»; elle inclut aussi les acquis de chercheurs des

${ }^{1}$ E. Kraskowska, «Wstęp », [dans :] W. Bolecki, E. Kraskowska (dir.), Kultura w stanie przekładu. Translatologia - komparatystyka - transkulturowość, Fundacja Akademia Humanistyczna — Instytut Badań Literackich PAN, Warszawa 2012, p. 9.

${ }^{2}$ La question de savoir dans quelle mesure ces textes ont été et sont actuellement cités mérite une étude approfondie ; elle est partiellement abordée dans une version polonaise élargie de la présente étude : M. Kaźmierczak, «Wieża Babel czy wieża z kości słoniowej? Recepcja przekładoznawstwa zachodniego w rosyjskojęzycznej nauce o przekładzie - próba rozpoznania ", Przektadaniec 41, 2020. 
pays du bloc socialiste, tels la Pologne ou la Tchécoslovaquie. La perspective des échanges d'idées entre les centres scientifiques forts qu'étaient les deux anciens blocs politiques s'en trouvera certes perturbée, mais les apports des Polonais et des Tchèques à la traductologie occidentale justifient assurément leur inclusion dans cette dernière. En revanche, les traductions de travaux publiés originellement en letton, lituanien et estonien ne seront pas prises en compte, venant de pays qui faisaient partie de l'espace culturel de l'URSS. De même, nous ignorerons les textes publiés en langue étrangère par des auteurs russes ou soviétiques et traduits ultérieurement.

Il n'existe pas encore de véritable méthodologie de recherche concernant les itinéraires qu'a empruntés la pensée traductologique via la traduction ${ }^{3}$. Elle n'est pas réellement abordée dans le recueil A History of Modern Translation Knowledge, où le sujet n'est qu'effleuré par Lieven D'hulst. Alexandra Assis Rosa a examiné les formes de diffusion des connaissances dans le domaine de la traduction, mais ne tient pas compte des traductions elles-mêmes ${ }^{4}$. Je me suis donc tournée vers les approches et remarques méthodologiques formulées dans les travaux de chercheurs tels que Elżbieta Skibińska, Anna Bednarczyk, Ewa Konefał, Natal'â Sokolova ${ }^{5}$ et Daniel Gile ${ }^{6}$, sans toujours partager entièrement leurs points de vue. Leurs travaux reposent souvent sur des bases telles que les bibliographies. Il n'existe toutefois, apparemment, aucun inventaire ou index des traductions russes de textes traductologiques. Les réflexions qui suivent s'appuieront donc sur une bibliographie dressée pour les besoins de mes études ${ }^{7}$.

3 Les itinéraires de la théorie de la littérature et des études culturelles ont été étudiées, notamment les «voyages » des concepts particuliers, voir par ex. : Ş. Susam-Sarajeva, Theories on the Move. Translation's Role in the Travels of Literary Theories, Brill, Amsterdam 2006. Ma méthodologie, cependant, est principalement axée sur une recherche métatraductologique et une tentative d'approche holistique des groupes de textes.

4 L. D'hulst, « Transfer modes », [dans :] L. D’hulst, Y. Gambier (dir.), A History of Modern Translation Knowledge. Sources, Concepts, Effects, John Benjamins, Amsterdam 2018, pp. 135-142 ; A. Assis Rosa, «Forms and formats of dissemination of translation knowledge », [dans :] A History of Modern Translation Knowledge..., pp. 203-213.

5 La norme de translittération utilisée dans cet article est l'ISO 9 de 1995.

6 E. Skibińska, « La traduction dans les revues comme voie de circulation des voix théoriques. L'exemple des revues polonaises Pamiętnik Literacki (1956-1994), Teksty (1972-1981) et Teksty Drugie (1990-2010) », [dans :] I. Génin, I. Klitgård (dir.), Translating the Voices of Theory / La Traduction des voix de la théorie, Éditions québécoises de l'œuvre, Montréal 2015, pp. 113-134; A. Bednarczyk, « Przekładoznawcze zmagania polskiej rusycystyki », [dans :] B. Stempczyńska, P. Fast (dir.), 50 lat polskiej rusycystyki literaturoznawczej, Uniwersytet Śląski, Katowice 2000, pp. 119-140 ; E. Konefał, « W poszukiwaniu metodologii badań metaprzekładoznawczych », Rocznik Przekładoznawczy 14, 2019, pp. 247-262 ; N. Sokolova, « Naukometričeskij podhod kak èffektivnyj instrument analiza sovremennogo sostoâniâ perevodovedeniâ », Vestnik MGU. Série 22 : Teoriâ perevoda 4, 2017, pp. 58-69 ; D. Gile, «Analyzing translation studies with scientometric data: from CIRIN to citation analysis », Perspectives. Studies in Translatology 23(2), 2015, pp. 240-248.

7 Cf. M. Kaźmierczak, op. cit. 
Le présent article n'est pas scientométrique à proprement parler : j'ai renoncé à une analyse détaillée de corpus au profit d'une approche transversale et donc moins systématique. Ma décision est motivée par les difficultés inhérentes à la constitution d'un corpus exhaustif ${ }^{8}$ : publications dispersées, difficulté de trouver les textes et de les classifier. Pour identifier les textes traduits, j'ai donc examiné le contenu de volumes collectifs, de revues de traductologie réputées à l'époque, et de revues professionnelles, scientifiques et culturelles récentes ; j'ai également puisé des informations dans des bases de données, catalogues de bibliothèques ${ }^{9}$, bibliographies de livres et de revues scientifiques. Cette collecte d'information n'a pas été facile, car les catalogues en ligne des bibliothèques russes ne couvrent pas la période antérieure à la première moitié des années 1990, et les bibliographies et notes de bas de page sont trompeuses quant à la nature des textes, car elles ne signalent pas les noms des traducteurs.

Les données recueillies, malgré toutes leurs imperfections, seront soumises à une étude qualitative dépassant la simple analyse bibliographique, qui devrait mettre en lumière les causes des phénomènes et tendances observés.

\section{PANORAMA DES PUBLICATIONS - $1^{\mathrm{e}}$ PARTIE $\left(2^{\mathrm{e}}\right.$ MOITIÉ DU XX ${ }^{\mathrm{e}}$ SIÈCLE)}

Je commence l'examen des traductions de publications de chercheurs occidentaux à partir de la deuxième moitié $\mathrm{du} \mathrm{XX}^{\mathrm{e}}$ siècle, époque où la traductologie a réellement vu le jour. Parmi les publications des années 1950 et 1960, j'ai pu trouver dix articles en version russe, dont six publiés dans Masterstvo perevoda ('L'art de traduire' ; désormais $M P$ ), principale tribune de réflexion sur la traduction littéraire en Union soviétique, et deux textes de Georges Mounin parus dans V zaŝitu mira $\left(\mathrm{n}^{\circ} 71\right.$ de 1957 et $\mathrm{n}^{0} 85$ de 1958), qui auraient été mieux à leur place dans $M P$. En effet, $V$ zasîtu mira, dont le titre de l'édition française était Horizons. La Revue de la Paix, n'était pas à proprement parler adapté à l'échange d'idées en traductologie, puisqu'il s'agissait d'une publication associée au Conseil mondial de la Paix, lequel dépendait fortement de $1^{\prime}$ Union soviétique ${ }^{10}$. Les deux derniers sont des articles de Yehoshua Bar-Hillel et de Victor Yngve sur la traduction

${ }^{8}$ Les problèmes de constitution des corpus sont abordés par Anthony Pym dans : Method in Translation History, Routledge, London-New York 2014, pp. 38-54 [1 ${ }^{\mathrm{e}}$ édition : 1998].

${ }^{9}$ Base de données de l'Institut d'information scientifique des sciences sociales de l'Académie des sciences de Russie (INION RAN), base de données BITRA de l'Université d'Alicante (sur environ 360 entrées indexant la langue russe, il n'y a pas de traductions); Bibliothèque d'État de Russie à Moscou, Bibliothèque nationale de Russie à Saint-Pétersbourg, bibliothèque numérique «CyberLeninka ».

10 Pierre Cot, homme politique français, en a été le rédacteur en chef. Publiée à Moscou de 1950 à 1961, la revue paraissait sous plusieurs titres. 
automatique et ont été publiés dans des revues, respectivement, de philologie et de cybernétique.

Parmi les premiers auteurs traduits, il y a ainsi des figures marquantes de l'histoire de la traductologie, tels Georges Mounin, ou Edmond Cary, co-fondateur de la Société française des traducteurs et de la revue internationale de traduction Babel. On retrouve aussi des thèmes qui ont donné naissance à des courants privilégiés : l'autoréflexion des traducteurs, en particulier lorsque ceux-ci étaient aussi des auteurs, comme Valéry Larbaud ou le poète polonais Julian Tuwim, ou la traduction automatique.

Pour les années 1970, j'ai trouvé douze travaux. Le texte-clé dans lequel Eugene Nida expose sa conception de la traduction a été publié dans la prestigieuse revue de linguistique Voprosy âzykoznaniâ $\left(n^{\circ} 4,1970\right)$ où il est confronté aux thèses de chercheurs russes (Efim Etkind conçoit la traduction littéraire comme « un art et une science », pp. 15-29). Masterstvo perevoda joue également un rôle majeur pendant cette décennie, en publiant neuf articles d'auteurs slaves, dont les Polonais Julian Tuwim et Andrzej Drawicz, le Bulgare Sider Florin, le Serbe Miodrag Sibinović et le Tchèque Jiří Levý : introduction de la dernière version de son livre Umění překladu ('L'art de traduire') (MP n ${ }^{0} 6,1969$ [1970]) $)^{11}$, après deux autres chapitres déjà publiés en 1966 [1968], accompagnés d'une présentation dans laquelle Bohuslav Ilek soulignait la contribution de son collègue prématurément décédé au développement de la discipline. Vient ensuite la revue littéraire Voprosy literatury ( $\left.\mathrm{n}^{\circ} 4,1970\right)$, qui publie des réflexions d'Aloys Skoumal, traducteur tchèque d'œuvres anglaises, précédemment parues dans la revue Babel et traduites du français ("Quelques remarques sur l'art de traduire », Babel $\mathrm{n}^{\mathrm{O}}$ 2(16), janvier 1970). Mis à part l'article de Nida, le seul texte véritablement occidental, traduit de l'italien, est celui de Clara Montella, qui présente ses observations sur les traductions russes d'une nouvelle de Boccace et les diverses stratégies qu'elles exploitent (Tetradi perevodčika $\left.n^{0} 15,1978\right)$. En outre, dans $M P$, on trouve une 'Conversation de citations' ( « Razgovor citat », $M P \mathrm{n}^{0}$ 7, 1970) de dix pages ; il s'agit d'une « collection » de passages de textes de 22 auteurs, principalement allemands (mais il y a aussi Alexander Tytler, Hilaire Belloc et José Ortega y Gasset), qui reprend l'annexe du livre Zielsprache. Theorie und Technik des Übersetzens (Zürich 1963) de Fritz Güttinger ${ }^{12}$.

À côté de ces articles, paraissent aussi des travaux de traductologie plus volumineux. L'on n'en trouve que six entre 1950 et 1980. Le premier (1957) est un recueil d'articles anglais sur la traduction automatique (W.N. Lock, A.D. Booth (dir.), Machine Translation of Languages, New York 1955). Cette publication précède, si je ne me trompe pas, les recherches soviétiques dans ce domaine, et

${ }^{11} M P$ a souvent été publié avec un certain retard. Dans ce cas, j'indique deux dates. Les volumes n'ont été numérotés qu'à partir du sixième tome, d'où les éventuelles divergences de description.

${ }^{12}$ Il n'est donc pas certain que les traductions aient été effectuées à partir des langues originales, ce qui constitue une exception dans les publications examinées. 
a apporté, entre autres, des réflexions de Bar-Hillel sur les idiomes. Vient ensuite un autre volume collectif sur le même sujet, traduit en russe à partir de plusieurs langues occidentales (Avtomatičeskij perevod, Moskva 1971), puis trois monographies de chercheurs slaves : le Tchèque Jiř́ Lévy, Uměni překladu (1974, texte intégral), le Slovaque Anton Popovič, Teória umeleckého prekladu (1975) et la Bulgare Anna Lilova, Uvod v obštata teorija na perevoda (1985).

À côté de ces ouvrages traduits intégralement, l'anthologie Voprosy teorii perevoda v zarubežnoj lingvistike ('Questions de théorie de la traduction en linguistique étrangère') de Vilen Komissarov ${ }^{13}$ occupe une place particulière. Les textes sont traduits de l'anglais, de l'allemand et du français, regroupés en sections thématiques, et leur ordre de présentation dans celles-ci n'est pas toujours chronologique. Le volume s'ouvre sur des questions de linguistique générale de la traduction : réflexions de Roman Jakobson sur les aspects linguistiques de la traduction (1966 [1959 $\left.]^{14}\right)$, article « Linguistic Analysis and Translation » de J.R. Firth (1956), début du livre Les problèmes théoriques de la traduction de Mounin (1963), extraits des ouvrages de M.A.K. Halliday (1966) et K.-R. Bausch (1971) traitant de linguistique contrastive, et approche de la traduction à la lumière de la théorie de la communication d'Otto Kade (1968). La section «Équivalence en traduction » reprend quatre chapitres de l'ouvrage de J.C. Catford A Linguistic Theory of Translation (1967), le chapitre «Principles of correspondence » de Towards a Science of Translating de Nida (1964), et des extraits de Translation und Translationslinguistik de Gert Jäger (1975). Dans la section « Processus de traduction », Komissarov met en perspective le classement des procédés (appelés en russe méthodes) de traduction proposé par Jean-Paul Vinay et Jean Darbelnet dans leur Stylistique comparée (1972 [1958]) et la notion de « Multiple Stage Translation» de Charles F. Voegelin (1954). La section « Pragmatique et stylistique de la traduction » réunit des travaux d'Albrecht Neubert (son article « Pragmatische Aspekte der Übersetzung », 1968) et de Katharina Reiss (sa typologie des textes en traduction, 1971). Comme le montre cette liste, il s'agit de textes qui demeurent aujourd'hui des références en traductologie.

Parmi les faits marquants de la réception russe d'ouvrages occidentaux dans les années 1980, il y a lieu de signaler deux articles, de James Holmes ${ }^{15}$ et d'André Lefevere (dans les actes du colloque Novye dostiženiâ v oblasti teorii perevoda ['Nouveaux acquis en théorie de la traduction'], 1978 ${ }^{16}$ ). Des textes d'auteurs étrangers sont également publiés aux côtés de travaux russes dans deux revues. Un volume intitulé 'La traduction en tant que moyen de rapprochement

13 V. Komissarov (dir.), Voprosy teorii perevoda v zarubežnoj lingvistike, trad. L. Černâhovskaâ, G. Turover, A. Batrak, Meždunarodnye otnošeniâ, Moskva 1978.

14 J'indique la date d'édition qui fait l'objet d'une traduction et, le cas échéant, j'ajoute la date de la première édition.

15 Texte anglais : «The Future of Translation Theory. A Handful of Theses », [dans :] Translated! Papers on Literary Translation and Translation Studies, Rodopi, Amsterdam 1988, pp. 99-102.

${ }^{16}$ N. Bažan et al. (dir.), Hudožestvennyj perevod. Voprosy teorii i praktiki, Izd. Erevanskogo un-ta, Erevan 1982. 
entre deux nations ${ }^{17}$ réunit ainsi les observations d'un auteur traduit (Gabriel García Márquez), de deux poètes et traducteurs italiens (Margherita Guidacci et Roberto Mussapi) et de Robert Daglish, traducteur de Mikhaïl Cholokhov. Les textes de Larbaud, Tuwim et Alfred Kurella présentés auparavant dans le numéro de $M P$ déjà évoqué y sont aussi repris, de même que des propos de Georges Mounin et Octavio Paz provenant du Courrier de l'UNESCO, publié dans différentes langues (les auteurs des différentes versions linguistiques n'étant pas clairement indiqués, elles sont considérées comme des originaux $)^{18}$. En réalité toutefois, la seule contribution théorique étrangère incluse dans ce volume est un texte du comparatiste Dionýz Durišin, sur sa conception structuraliste. Le second volume, consacré à la poétique de la traduction ${ }^{19}$, reprend des propos du traducteur tchèque Ewald Osers, du théoricien slovaque František Mika, et d'Artur Sandauer, critique et traducteur polonais. L'article d'Osers sur la traduction poétique, publié à l'origine dans le volume Hudožestvennyj perevod. Voprosy teorii i praktiki de 1982, paraît ici dans une version élargie et dans une nouvelle traduction, et l'on peut donc dire que la réflexion traductologique qu'il contient aura d'abord été publiée en russe dans une série de traductions.

Pour les années 1990, j'ai trouvé deux textes, dans des volumes collectifs. Le premier — des réflexions d'Antonio Pamies Bertrán sur le rôle du rythme (1996) - confirme une préférence pour les contributions traitant de traduction poétique. Le second a davantage de poids en termes de circulation des théories : il s'agit d'un troisième texte de Nida, sur l'isomorphisme et l'équivalence (1997).

Des réflexions sur la traduction apparaissent également dans les éditions traduites d'ouvrages de Humboldt, Herder, Goethe, Montesquieu, Willard Quine ou autres philosophes qui se sont intéressés à la langue. Par exemple, l'essai de José Ortega y Gasset Miseria y esplendor de la traducción est disponible en russe depuis 1991.

\section{AUTRES FORMES DE TRANSFERT POSSIBLES}

Compte tenu du nombre apparemment restreint de traductions publiées au $\mathrm{XX}^{\mathrm{e}}$ siècle, la question se pose de savoir s'il y a quelque chose qui puisse les remplacer. Je ne présenterai ici que quelques-uns des substituts possibles ${ }^{20}$. Il y a par exemple les critiques de travaux traductologiques étrangers et les présentations de revues occidentales de traduction. Grâce aux longues listes bibliographiques

17 A. Klyško (dir.), Perevod - sredstvo vzaimnogo sbliženiâ narodov. Hudožestvennaâ publicistika, Progress, Moskva 1987.

$18 C f$. aussi : G. Mounin, "Le traducteur entre les mots et les choses », Le Courrier de l'UNESCO 4, 1962, pp. 25-28 ; O. Paz, « Sobre la traducción », El Correo de la UNESCO 2, 1975, pp. 36-40.

19 S. Gončarenko (dir.), Poètika perevoda, Raduga, Moskva 1988.

20 J'y reviens plus en détail dans : M. Kaźmierczak, op. cit. 
publiées par Masterstvo perevoda, parfois accompagnées de commentaires, les lecteurs savaient au moins ce qui se publiait ailleurs. Les idées considérées comme importantes étaient évoquées et commentées. Vladimir Samsonov a ainsi vérifié les bases de la thèse de l'indétermination de la traduction de Quine ${ }^{21}$. Le manuel de Komissarov écrit au tournant des $\mathrm{XX}^{\mathrm{e}}$ et $\mathrm{XXI}^{\mathrm{e}}$ siècles et consacré à la théorie générale de la traduction du point de vue des « savants étrangers ${ }^{22}$ a été le premier livre à dresser un panorama des théories occidentales. Reposant sur 54 références bibliographiques en cinq langues, il présente brièvement les apports par régions géopolitiques ${ }^{23}$ : Angleterre, France et Canada, États-Unis, Allemagne de l'Est, Allemagne de l'Ouest et — en s'appuyant sur les publications en anglais - Scandinavie. Le dernier chapitre est consacré à Güttinger, Aleksandr Ludskanov et Gideon Toury. Les publications ultérieures du même type - parues au $\mathrm{XXI}^{\mathrm{e}}$ siècle - sont de plus en plus nombreuses et restent dans la lignée des travaux de Komissarov.

Les ouvrages de chercheurs étrangers écrits en russe contribuent également à la diffusion des approches originaires d'autres pays perçues comme théories itinérantes. Parfois, il est difficile de les distinguer des traductions, car les bibliographies et les citations russes omettent systématiquement les noms des traducteurs des travaux de traductologie. Dans le corpus examiné, c'est le cas, entre autres, des auteurs qui parlent russe et pourtant sont traduits, comme Kade, Neubert, Lilova ou Kinga Klaudy. La réflexion traductologique étrangère est importée dans la recherche russe de diverses manières. Il s'agit donc de phénomènes de transfert (selon Lieven $\mathrm{D}^{\prime}$ huls $\mathrm{t}^{24}$ ) dépassant la traduction, avec toutes les difficultés que cela implique pour la recherche.

\section{PANORAMA DES PUBLICATIONS - 2 $2^{\mathrm{e}}$ PARTIE (XXI ${ }^{\mathrm{e}}$ SIÈCLE)}

L'analyse de la réception éditoriale au $\mathrm{XX}^{\mathrm{e}}$ siècle montre que l'épanouissement de l'activité de traduction en Union soviétique ${ }^{25}$ ne s'est pas accompagné d'un accroissement du nombre de traductions d'ouvrages de traductologie. Même

21 V. Samsonov, « K analizu gipotezy Kuajna o neopredelënnosti perevoda », Tetradi perevodčika 16, 1979, pp. 21-29.

22 V. Komissarov, Obŝâ̂ teoriâ perevoda. Problemy perevodovedeniâ v osvểenii zarubežnyh učenyh, ČeRo, Moskva 1999. Réimpression intégrale dans : V. Komissarov, Sovremennoe perevodovedenie. Učebnoe posobie, ÈTS, Moskva 2001/2004, pp. 179-316.

${ }^{23}$ Pour une définition des champs de recherche en histoire de la traduction basée, par ex., sur les critères géopolitiques, voir : J. St André, « History », [dans :] M. Baker, G. Saldanha (dir.), The Routledge Encyclopedia of Translation Studies, Routledge, London 2009, p. 134.

${ }^{24}$ L. D'hulst, op. cit.

25 À propos de ce phénomène connu, voir entre autres : L. Nelûbin, G. Huhuni, Nauka o perevode. Istoriâ i teoriâ s drevnejšì vremen do naših dnej, $4^{\mathrm{e}}$ édition, Flinta, Moskva 2018, pp. 321-325 ; V. Komissarov, « Russian Tradition », [dans :] M. Baker, K. Malmkjær (dir.), Routledge Encyclopedia of Translation Studies, Routledge, London-New York 1998, pp. 545-546. 
si je n'ai pas trouvé toutes les traductions, il ne fait pas de doute qu'elles ont été peu nombreuses. Un regard critique de l'intérieur sur les textes concernés le confirme. Pavel Toper (en se référant à Holmes, qui juge nécessaire la réception de la traductologie soviétique en Occident) admet qu'il est très rare que les ouvrages des auteurs étrangers soient cités, et que leurs traductions russes sont « quasi inexistantes ${ }^{26}$. Il convient de vérifier si ce diagnostic formulé en 2001 est toujours valable aujourd'hui.

Voyons d'abord les neuf livres importants que j'ai trouvés. Chronologiquement parlant, la publication en russe de l'ouvrage de Jan de Waard et d'Eugene Nida sur la traduction biblique, From One Language to Another. Functional Equivalence in Bible Translating, 1986 (Saint-Pétersbourg 1998), autrefois inconcevable ${ }^{27}$, peut certainement être considérée comme le début d'une nouvelle époque. D'un côté, les Russes rattrapent des années de retard en accueillant $A$ Linguistic Theory of Translation de Catford, 1967 (Moskva 2004), dont seuls certains passages avaient été traduits, l'essai de Jacques Derrida Des Tours de Babel, 1985 (Saint-Pétersbourg 2002) ou After Babel de George Steiner, 1975 (Moskva 2020), un incontournable. D'un autre côté, des traductions d'ouvrages contemporains paraissent, peu après la publication de leur première édition ou de l'édition mise à jour qui a servi de base à la traduction. Ce paramètre est très important, car comme le souligne D'hulst, la traduction de nouveaux travaux scientifiques joue un rôle crucial dans la diffusion des idées ${ }^{28}$. Si l'on pouvait considérer les versions russes de Entwicklungslinien der Translationswissenschaft d'Erich Prunč (Moskva 2015) et de Exploring Translation Theories d'Anthony Pym (Saint-Pétersbourg 2018) comme l'annonce de traductions systématiques à venir, elles pourraient être la manifestation d'une certaine politique de publication ; mais il est encore trop tôt pour en juger. Il convient également de souligner deux choses : tout d'abord, les publications de la première décennie du $\mathrm{XXI}^{\mathrm{e}}$ siècle - celles de Derrida, Catford, et aussi Eco et Robinson dont nous allons parler plus loin — ont vite été réimprimées (ce qui, indépendamment du public restreint de ces publications, s'explique aussi par leur petit tirage initial, de 2000 à 4500 exemplaires) ; ensuite, les éditeurs des traductions des livres de Prunč et Pym ont soumis celles-ci à la lecture critique de spécialistes du domaine. La récente publication en russe du livre Is That a Fish in Your Ear de David Bellos (Moskva 2019) semble cependant indiquer que les éditeurs ne dédaignent pas les traductions de textes à caractère de vulgarisation.

Parmi les traductions publiées après 2000, on trouve aussi des articles. Après la disparition de nombreuses revues réputées de l'époque soviétique (le treizième

26 P. Toper, Perevod v sisteme sravnitel'nogo literaturovedeniâ, Nasledie, Moskva 2001, p. 20.

27 Anna Bednarczyk note (dans : Zmagania z przekładem w przestrzeni rosyjskojęzycznej. Teoria i praktyka w ewolucji, Wydawnictwo Uniwersytetu Gdańskiego, Gdańsk 2016, p. 96) que la possibilité d'aborder la problématique de la traduction des textes religieux est l'un des grands changements survenus dans la traductologie russe après 1990.

28 L. D’hulst, op. cit., p. 139. 
et dernier tome de Masterstvo perevoda, daté de 1985, est sorti en 1990), des périodiques de profils variés ont occupé le créneau. Tout comme dans les décennies précédentes, les traductions de textes de traductologie sont parues aussi dans des revues non spécialisées dans ce domaine. En raison de cette dispersion, ainsi que du nombre croissant des revues scientifiques et culturelles, les données que j'ai recueillies ne sont probablement pas exhaustives. Elles permettent toutefois de signaler plusieurs phénomènes.

La traduction des travaux de Friedrich Schleiermacher (« Über die verschiedenen Methoden des Übersetzens », Vestnik MGU. Série 9, n 2, 2000), Paul Ricœur (« Le paradigme de la traduction », 2000 ; « Défi et bonheur de la traduction », Logos $n^{0} 5-6,2011$ ) ou Walter Benjamin (« Die Aufgabe des Übersetzers ») prouve que l'intérêt pour la traductologie européenne et le contexte philosophique de la traduction est bien ancré. Concernant l'ouvrage de ce dernier, nous avons même affaire à une série de traductions presque synchrones (quatre à ma connaissance : deux en 2000, 2004, 2012) qui sont également devenues le point de départ d'une réflexion métacritique, puisque deux d'entre elles ont fait l'objet d'une analyse approfondie d'Igor Čubarov ${ }^{29}$. En revanche, les conceptions de Quine sont un exemple d'assimilation rendue possible par les discussions dont elles ont fait l'objet (voir ci-dessus). En outre, les deux traductions de son article « Indeterminacy in Translation Again » de 1987 peuvent également être confrontées au texte original, qui les accompagne $\left(\operatorname{Logos} \mathrm{n}^{\circ} 2,2005 \text {, pp. } 28-41\right)^{30}$.

L'apport du bimestriel Logos — en particulier le numéro 5-6 de 2011 - mérite d'être souligné. Il contient, entre autres, l'introduction et le troisième chapitre du livre d'Antoine Berman L'Épreuve de l'étranger (Paris 1984) et la polémique d'Henri Meschonnic et Jean-René Ladmiral (« Poétique de... / Théorèmes pour... la traduction ») qui a ouvert en 1981 le numéro thématique de Langue française consacré à la traduction. On y trouve également des textes de Derrida, Ricœur et Michaël Oustinoff (ainsi qu'une interview de ce dernier). Logos est cependant une revue philosophique et littéraire, ce qui détermine une certaine spécificité des textes qu'elle publie occasionnellement sur la traduction, le choix des auteurs publiés, et donc le faible impact de ses traductions dans le milieu des traductologues ${ }^{31}$.

Une série de périodiques scientifiques prestigieux publiés par l'Université de Moscou (Vestnik MGU. Série 22), consacrées à la théorie de la traduction forment en revanche un forum de discussion strictement réservé aux spécialistes

${ }^{29}$ I. Čubarov, « Perevod kak opyt nečuvstvennyh upodoblenij. Pričiny neudač perevodov Zadači perevodčika Val'tera Ben'âmina na russkij âzyk », Logos 5-6 (84), 2011, pp. 237-252.

${ }^{30}$ La traduction du livre Word and Object, dont la thématique dépasse les questions de traduction, a également été publiée (Moskva 2000).

31 À titre d'exemple, selon CyberLeninka, un texte aussi important que l'extrait de L'épreuve de l'étranger de Berman n'est cité que 13 fois, alors que près d'une décennie s'est écoulée depuis sa publication en Russie (<https://cyberleninka.ru> [consulté le 29.01.2020]). 
du domaine. La création en 2008 d'une revue traductologique parmi les revues de cette université a été un signe d'émancipation de la discipline. La rédaction s'est déclarée prête à accueillir les « voix extérieures » ${ }^{32}$ et s'est engagée à publier les propos des chercheurs étrangers actuels, généralement en version bilingue langue étrangère-russe (les deux versions étant parfois de l'auteur même). Dès son premier numéro, le périodique s’est assuré la contribution de Michel Ballard (« Éléments pour une méthodologie réaliste en traductologie », Vestnik MGU. Série 22, $n^{0}$ 1, 2008 ; suivi de « Proverbe et traduction », $\left.n^{\circ} 2,2009\right)$, dont 1'ouvrage sur l'unité de traduction a en outre été publié à Irkoutsk (2009). La contribution de Vestnik $M G U$ à la circulation des idées occidentales comporte également des bilans de travaux de traductologues réputés, et les traductions publiées sont révisées par des rédacteurs scientifiques.

Malgré la consolidation de la discipline, les travaux de traductologie sont souvent publiés dans des revues de philologie, linguistique, études littéraires ou autres domaines ; ils sont tantôt rassemblés dans des numéros thématiques, tantôt dispersés. Parmi ces textes, on peut également retrouver des traductions. Citons comme exemple un volume publié par les éditions de l'Université de Saint-Pétersbourg, Vestnik SPbGU (série 'Philologie, études orientales, journalisme') consacré à la traduction, où sont publiées les traductions de textes ${ }^{33}$ de quatre représentants de différents centres académiques et méthodologies de recherche : Edward Balcerzan, Susan Bassnett, Yves Gambier et Nike Pokorn (Pologne, Grande-Bretagne, Finlande/France, Slovénie).

Nous ne pouvons pas ignorer dans cette analyse la revue de traducteurs Mosty ('Ponts'), à vocation à la fois pratique — tenant compte de l'activité professionnelle et du marché de la traduction actuels — et de réflexion scientifique. En 17 ans depuis 2004 (68 numéros), Mosty a publié six textes répondant aux critères adoptés dans notre travail. L'article de Cathy Flick, paru dans le premier numéro, dévoile l'approche actuelle et le pragmatisme de la rédaction : l'auteure y recueille les questions les plus fréquemment posées par les clients aux traducteurs (souvent avec un certain ton de reproche) et les réponses de ces derniers. À son tour, l'essai d'Hilaire Belloc $\left(\right.$ Mosty $\left.n^{\circ} 2,2013\right)$ s'inscrit dans le courant « écrivains étrangers s'exprimant sur l'art de traduire », déjà évoqué. L'autoréflexion de Manfred Frühauf sur la traduction de la poésie chinoise en allemand (Mosty $\left.n^{0} 3,2016\right)$ est un cas rare de publication de texte dont la thématique n'est pas associée au contexte russe. Signalons que les interviews de Jacolyn Harmer et Laura Burian, interprètes simultanées et enseignantes au Middlebury Institute of International Studies de Monterey, Californie (Mosty $n^{0} 1$, 2017) sont aussi des traductions. Vient ensuite un texte qui a déclenché une polémique : il traite des thèses discutables du journa-

32 N. Garbovskij, [Ot glavnogo redaktora], Vestnik MGU. Série 22, no 1, 2008, p. 4.

33 Il n'y a pas de premières éditions signalées dans les langues indiquées comme langues sources, à savoir le polonais et l'anglais. 
liste américain Joel Garreaux sur les perspectives de la traduction automatique ${ }^{34}$ et les confronte à une évaluation objective réalisée par des spécialistes russes ${ }^{35}$. Vient enfin la traduction d'extraits de l'ouvrage de Danica Seleskovitch et Marianne Lederer Interpréter pour traduire de 1984 publiés dans le numéro 3 de 2018 de Mosty. Ces ouvrages contribuent largement à l'intégration des courants de recherche occidentaux.

Passons maintenant aux aspects traductologiques des publications consacrées à la pratique de la traduction. En 2008, parait l'édition russe de la brochure de l'Union européenne sur les nouvelles technologies de traduction (Human Language Technologies for Europe, 2006, chef de projet [sic] Gianni Lazzari). Vient ensuite le périodique Professional'nyj perevod i upravlenie informaciej ('La traduction professionnelle et la gestion d'informations') (2008-2016), initiative intéressante qui contient des traductions de textes tirés de MultiLingual Computing $\&$ Technology et de Tcworld. Parmi leurs auteurs, nous retrouvons des chercheurs connus comme Hanna Risku (Professional'nyj perevod i upravlenie informaciej $\mathrm{n}^{\mathrm{o}} 6(18)$, juillet 2008), qui écrivent cependant cette fois en qualité de praticiens ; la traduction de leurs articles ne contribue donc pas à la circulation de la théorie, mais au transfert du savoir et des compétences pratiques.

Vient enfin le mensuel culturel et littéraire Inostrannaâ literatura ('Littérature étrangère') fondé en 1955. Sa principale vocation est de publier des œuvres littéraires traduites de différentes langues : à l'origine, il était pour le lecteur soviétique une des rares possibilités de découvrir la littérature mondiale autrement inaccessible. Parfois, cependant, les textes étaient accompagnés d'une réflexion du traducteur ou d'importants essais sur la traduction. Parmi les deux types de discours, on peut trouver des traductions (certains textes publiés dans le volume Perevod - sredstvo vzaimnogo sbliženiâ narodov de 1987, comme celui de Daglish, proviennent de la section 'La Tribune du traducteur'). Bien que le périodique n'ait pas pour vocation de publier des articles scientifiques, les textes parus dans les années 2017-2019 complètent de manière intéressante l'ensemble présenté ci-dessus. Sur cette période, on voit paraître l'essai de Susan Sontag « The World as India » et le discours prononcé en 1985 par Heinrich Böll à l'occasion de l'ouverture du Collège européen des Traducteurs de Straelen (Inostrannaâ literatura $\mathrm{n}^{0} 7$ et 12,2017). Ce choix de textes s'inscrit dans la lignée des genres et thèmes qu'abordait Masterstvo perevoda, relevant d'un discours très général sur la traduction. L'article du slaviste français Georges Nivat sur la relation entre la poésie étrangère, la traduction et la poésie originale (Inostrannaâ literatura $\mathrm{n}^{0} 7$,2018) relève en revanche du discours scientifique. En 2019, Inostrannâa literatura publie ( $\mathrm{n}^{\mathrm{o}} 4$ et 9 ) des chapitres de deux livres : l'un de Bellos (comme

34 Texte original : J. Garreaux, « Tongue in Cheek », Washington Post, 24 mai 2009.

35 M. Orël, « Stop, mašina! »; M. Cvilling, « Net povesti zaputannej v prirode, čem povest ${ }^{\prime}$ o mašinnom perevode »; I. Knižnik, « Vzglâd i nečto », Mosty 4(24), 2009. 
annonce d'une publication intégrale, voir ci-dessus), et l'autre, philosophique, de Ricœur («'Un passage' : traduire l'intraduisible » — la troisième partie de ses réflexions Sur la traduction, qui ont été intégralement traduites en russe, mais dans des publications dispersées).

$\mathrm{Au}$ total, les ouvrages traduits ces deux dernières décennies sont beaucoup plus nombreux que pendant tout le demi-siècle précédent. Leur nombre croissant et leur diversité permettent de constater que les textes traitant de traduction (pas nécessairement scientifiques) connaissent un véritable essor.

\section{MOTIVATION DE LA TRADUCTION (OU DE L'ABSENCE DE TRADUCTION)}

Examinons maintenant pourquoi, au vu du foisonnement de publications traductologiques actuelles, certaines sont traduites pour le lecteur russe et d'autres pas. Cela ne remet évidemment pas en cause la pertinence des textes ni les choix des éditeurs, rédacteurs et traducteurs, mais il est indéniable que les œuvres qui méritent d'être importées sont toujours nombreuses, et certains facteurs doivent donc déterminer les choix.

Depuis la réception des ouvrages de Cary et Nida jusqu'à la traduction des travaux de Pym et Bassnett, l'importance du chercheur, la portée de ses découvertes et de ses propositions théoriques ont sans aucun doute été prises en compte. Le critère de fond est certainement à l'origine des choix de Komissarov pour son anthologie de 1978 (voir point 2), et le développement de la discipline a confirmé la validité de ce critère dans la plupart des cas. D'autre part, la précision indiquée dans le titre ( « ... en linguistique étrangère ») révèle une certaine limitation : celle imposée par le paradigme linguistique de la recherche traductologique longtemps privilégié en Russie, dont la domination — également idéologique — n'était pas propice à l'importation d'ouvrages présentant des méthodologies nettement différentes ${ }^{36}$.

La relation entre le texte sélectionné pour être traduit et la langue et la culture russes est également un facteur important. Dans les ouvrages recueillis, ce critère est rempli par un pourcentage très important d'œuvres relevant de la critique de la traduction et de l'autocommentaire. Au vu des proportions observées, on peut supposer que la finesse et l'humour de l'analyse ne sont pas les seuls éléments à avoir ouvert la voie pour l'article «Czterowiersz na warsztacie » ('Quatrain en gestation') (MP, 1964 [1965]) de Julian Tuwim : avant tout, le poète-traducteur polonais décortique le début de Rouslan et Ludmila d'Alexandre Pouchkine. Le fait que ses considérations n'ont pas été incluses dans la section 'Questions de traduction à l'étranger' (à côté de Cary et Larbaud), mais dans la rubrique

\footnotetext{
36 À propos des conditions idéologiques de réception des théories, voir Ş. Susam-Sarajeva, op. cit.
} 
'Pouchkine multilingue' le confirme bien. On peut probablement en dire autant de la sélection ultérieure de l'article d'Andrzej Drawicz (MP n ${ }^{\circ} 11,1976$ [1977]) qui a examiné les traductions du polonais faites par les excellents poètes russes Akhmatova et Pasternak ${ }^{37}$. Le texte d'Olga Uličná ( $M P \mathrm{n}^{0} 10,1974$ [1975]) traite de la réception de Pouchkine en Tchécoslovaquie. Ewald Osers était un traducteur anglophone de la littérature d'Europe centrale, en particulier de la poésie tchèque, mais il traduisait également de l'allemand, du macédonien et des dialectes lachs. Le succès de son texte en russe (deux versions publiées respectivement en 1982 et 1988, voir point 2 ci-dessus) a été certainement déterminé par le choix du sujet : les difficultés de la traduction de la poésie russe en anglais.

La motivation associée à la culture cible est encore plus clairement illustrée par deux traductions du français. Dans son texte publié dans $M P$ en $1963^{38}$, d'abord paru dans Babel $^{39}$, André Meynieux présente l'anthologie des publications des écrivains russes sur la traduction ${ }^{40}$ de Ûrij Levin et Andrej Fëdorov. Il apprécie beaucoup cet ouvrage et le présente comme un exemple d'initiative éditoriale digne d'être suivie dans d'autres pays. Cette traduction de la critique étrangère - un type de texte rarement traduit — est donc à comprendre comme un retour d'information très flatteur pour les rédacteurs et un motif de satisfaction pour les lecteurs. Un autre exemple de «transfert à rebours » de réception d'ouvrage enthousiaste date de 2018, avec la publication de l'article de Nivat dans Inostranna â literatura. L'auteur y parle de la tradition russe de traduction de poésie, la voit comme un phénomène unique et constate qu'en Russie, la traduction, par son engagement éthique, est toujours « plus qu'une traduction». On peut penser que pour le lecteur cible, ce n'est pas le contenu objectif de l'article qui compte (il présente un aperçu concis de l'activité de traducteur de fameux écrivains de différentes époques, chose que l'on retrouve dans d'autres publications), mais l'admiration non dissimulée du philologue slavisant français pour le pouvoir d'assimilation de la culture russe et son évaluation élogieuse de l'école soviétique de traduction.

Les textes d'auteurs étrangers qui se proposent de faire un tour d'horizon traductologique constituent une autre catégorie privilégiée. Ils présentent une valeur certaine pour découvrir dans les grandes lignes l'histoire de la traduction ou de la pensée traductologique de certains pays (pour la France, Cary, MP, 1964 [1965]),

${ }^{37}$ Fait significatif, on a recouru à un texte originellement publié dans un volume explorant les relations culturelles polono-soviétiques : A. Drawicz, « Anna Achmatowa i Borys Pasternak jako tłumacze liryki polskiej », [dans :] B. Galster, K. Sierocka en coopération avec A. Piorun (dir.), Po obu stronach granicy. Z powiazań kulturalnych polsko-radzieckich $w$ dwudziestoleciu międzywojennym, IBL — ZS PAN, Wrocław 1972, pp. 163-179.

38 A. Men'ë, « Primer, dostojnyj podražaniâ », trad. M. Lorie, MP, 1963, pp. 225-230.

39 A. Meynieux, « Un exemple à suivre. Iou.D. Lévine et A.V. Fëdorov : Les écrivains russes parlent de la traduction $\left(\mathrm{XVII}^{\mathrm{e}}-\mathrm{XX} \mathrm{e}^{\mathrm{e}}\right.$ siècles) », Babel 7(2), 1961, pp. 85-87.

40 U. Levin, A. Fëdorov (dir.), Russkie pisateli o perevode: XVIII-XX vv., Sovetskij pisatel', Leningrad 1960. 
certaines spécialisations (traducteurs militaires français - Ramuntxo Gardères, Vestnik MGU. Série 22, $\mathrm{n}^{\mathrm{O}} 2$ 2, 2010), certaines périodes (la traduction au Moyen Âge par Astrid Guillaume ${ }^{41}$, texte de Bassnett sur le développement des Translation Studies depuis $1975^{42}$ ). L'intégration des approches occidentales n'est pas uniquement due aux propos du chercheur occidental lui-même, mais également au fait que les textes sélectionnés pour la traduction sont des métanarrations qui familiarisent le lecteur à l'histoire et aux perspectives du domaine : c'est aussi le cas des livres de Prunč et de Pym. Pour le chercheur autrichien, il est à remarquer que le titre de son ouvrage a été retouché : il précise « la voie du développement de la réflexion traductologique occidentale » (cf. Entwicklungslinien der Translationswissenschaft. Von den Asymmetrien der Sprachen zu den Asymmetrien der Macht - Puti razvitiâ zapadnogo perevodovedeniâ. Ot âzykovoj asimmetrii k političeskoj), ce qui, d'une part, fournit aux destinataires des informations importantes sur le contenu de l'ouvrage (qui évoque d'ailleurs aussi certaines conceptions russes), mais d'autre part peut suggérer des relations linguistiques et politiques asymétriques, mentionnées dans le sous-titre, dont le contexte russe est exclu.

Les textes étrangers de caractère populaire ou pragmatique intéressent également les traducteurs, ou peut-être surtout, les éditeurs. Trois livres parus au XXI siècle peuvent être classés dans cette catégorie. Dire quasi la stressa cosa d'Umberto Eco présente clairement les phénomènes de traduction en partant de l'expérience de l'auteur (célèbre) traduit, ce qu'exprime son sous-titre : Esperienze di traduzione. Le caractère pragmatique se reflète dans le titre même du manuel de Douglas Robinson, Becoming a Translator, et davantage encore dans sa version russe 'Comment devenir traducteur' (Kak stat' perevodčikom, 2005). Le péritexte du livre de Bellos (tout comme la note qui a accompagné la publication de ses extraits) indique qu'il est adressé à un public qui s'intéresse à la traduction sans être professionnel du domaine.

Les facteurs idéologiques aussi ont leur importance. À l'époque de l'Union soviétique, les conditions politiques favorisaient les courants de recherche originaires plutôt d'Allemagne de l'Est que de la République fédérale d'Allemagne (École de Leipzig vs Théorie du skopos). Les sympathies politiques de certains auteurs pouvaient aussi avoir une incidence sur leur publication. On peut ainsi se demander si la réimpression en 1987 de la traduction du discours d'Alfred Kurella, prononcé en 1954 lors du Congrès de l'Association des Écrivains de la RDA à Berlin, est réellement due à la pertinence de ses thèses (l'idée de développer la théorie de la traduction), ou plutôt à la position de l'écrivain, militant

41 A. Gijom, « Perevod v Srednie veka: čeharda smyslov » / « La Traduction médiévale sens dessus dessous », trad. D. Balandina, Û. Koreneva, rédactrice scientifique O. Kostikova, Vestnik MGU. Série 22, nº 1, 2011, pp. 38-66.

42 S. Bassnett, « Istoki i razvitie perevodovedeniâ v 1975-2016 gg » [The origins and development of Translation Studies 1975-2016], trad. T. Kazakova, Vestnik SPbGU. Série 9, n 4, 2016, pp. 31-44. 
communiste et co-auteur de la politique culturelle de la $\mathrm{RDA}^{43}$. L'appartenance de Mounin au Parti communiste français pourrait aussi expliquer en partie la réception relativement importante de ses textes. Ou encore, la diffusion des instructions de Friedrich Engels sur la façon de ne pas traduire les écrits de Marx ${ }^{44}$ a de toute évidence un caractère politique.

Les contacts institutionnels, enfin, sont un facteur favorable. La réception de l'article de Gambier (Vestnik $S P b G U$, voir ci-dessus), affilié à l'Université de Turku, a bénéficié de la proximité géographique de Saint-Pétersbourg et des liens entre les centres universitaires finlandais et russes. Pour nous référer à la remarque de D'hulst ${ }^{45}$, le transfert des idées est ici favorisé par les déplacements du chercheur.

Revenons sur les raisons du nombre limité de traductions au $\mathrm{XX}^{\mathrm{e}}$ siècle. J'ai déjà mentionné les interdictions politiques qui avaient entravé, notamment, la réception des théories relatives à la traduction biblique, des travaux de chercheurs de certains pays ou des courants qui ne cadraient pas avec le courant réaliste dominant, y compris en traduction et traductologie. La fierté des succès indéniables de la traductologie en langue maternelle, renforçant le sentiment de son autosuffisance, a pu être un autre facteur idéologique (dans les publications examinées, elle transparait clairement dans la critique des Problèmes théoriques de la traduction de Mounin par Avramov ${ }^{46}$ ). La revue Tetradi perevodčika ( ${ }^{\circ}$ Cahiers du traducteur', plus académique et plus complète sur le plan thématique que $M P$, à vocation artistique) était simplement nationale : aucune volonté d'assimiler des théories étrangères n'est exprimée par les éditeurs, et dans le résumé publié à l'occasion du vingtième anniversaire de la revue, seules des monographies russes ${ }^{47}$ sont mentionnées en tant que retards de révision à rattraper. Les 25 tomes de la revue (publiée avec des interruptions de 1963 à 2005) n'ont apporté qu'une seule traduction, l'article déjà évoqué de Montella, en $1978^{48}$.

Une autre raison de cette absence de traductions tient peut-être dans la conviction (des éditeurs, des décideurs ?) que les spécialistes de la traduction n'ont pas besoin de traductions de travaux théoriques parce qu'ils connaissent les langues étrangères. Cependant, la pratique démontre que le " multilinguisme passif »

43 Voir A. Kurella, « Teoriâ i praktika perevoda », trad. M. Taner, révision M. Lorie, MP, 1959, pp. 407-437. Réimpression dans : A. Klyško (dir.), Perevod - sredstvo vzaimnogo sbliženiâ narodov, Progress, Moskva 1987, pp. 106-131.

${ }^{44}$ F. Ėngel's, « Kak ne sleduet perevodit' Marksa » [traduit de l'anglais], [dans :] A. Klyško (dir.), Perevod..., pp. 283-291. Dans ce cas, l'absence de mention du traducteur est typique des éditions des classiques du marxisme-léninisme.

${ }^{45}$ L. D'hulst, op. cit., p. 136.

46 V. Avramov, «O knige Ž. Munèna Teoretičeskie problemy perevoda », Tetradi perevodčika 3 , 1966, pp. 103-104.

${ }^{47}$ Û. Vannikov, «Teoriâ perevoda v Tetradâh perevodčika », Tetradi perevodčika 20, 1983, p. 23.

48 Je l'examine plus à fond dans : M. Kaźmierczak, op. cit. 
qu'on leur impute est peu réel ${ }^{49}$. Le corpus que j'ai examiné illustre des phénomènes éloquents à ce propos - je ne les ai pas abordés faute de place - tels que le fait que la critique de la revue bilingue Meta n'est fondée que sur des documents en français ${ }^{50}$, ou les citations indirectes, qui prouvent qu'un philologue italien, par exemple, peut avoir besoin d'une traduction de l'anglais.

Les conséquences de l'absence de traductions dans le circuit peuvent être doubles. Soit les traductologues s'enferment dans une philologie donnée - ils s'en tiennent aux ouvrages écrits dans une langue étrangère donné ${ }^{51}$, soit tous sont obligés de publier dans une langue de grande diffusion, dont le russe fait partie. Aucune de ces perspectives n'est de bon augure pour le développement de la discipline $^{52}$ sur le plan local ou mondial, et il nous reste à espérer que la tendance visible à une augmentation des traductions se poursuive.

\section{CONCLUSIONS ET PERSPECTIVES}

Le corpus examiné permet de constater qu'au $\mathrm{XX}^{\mathrm{e}}$ siècle, les traductions russes d'ouvrages de traductologie ont été peu nombreuses et que dans la plupart des cas, il s'agissait de travaux d'auteurs slaves. L'importation des acquis de la traductologie occidentale sensu stricto s'est intensifié après le changement de régime, mais pas immédiatement ${ }^{53}$. En effet, c'est seulement dans la dernière décennie que nous pouvons observer une activité éditoriale accrue dans ce domaine, tandis que l'on ne traduit plus à partir des langues slaves (une seule publication enregistrée). Les textes traduits en russe relèvent cependant surtout de la vulgarisation scientifique ou traitent de traduction au sens large, sans contribuer

49 Passive multilingualism ; concept emprunté à M. Snell-Hornby, « Is Translation Studies going Anglo-Saxon? Critical comments on the globalization of a discipline », [dans :] D. Gile, G. Hansen, N.K. Pokorn (dir.), Why Translation Studies Matters, John Benjamins, AmsterdamPhiladelphia 2010, p. 100.

50 V. Rozencvejg, «Žurnal kanadskih perevodčikov Meta », Tetradi perevodčika 20, 1985, pp. 91-102.

51 Pour la situation en Pologne, voir J. Żmudzki, « Problemy, zadania i wyzwania translatoryki », Lingwistyka Stosowana 1, 2009, pp. 50-51, ou E. Skibińska, « Between Richness and "Non-existence". Polish Translation Researchers as a Community », [dans :] K. Taivalkoski-Shilov, L. Tiittula, M. Koponen (dir.), Communities in Translation and Interpreting, Éditions québécoises de l'œuvre, Montréal 2017, p. 261.

$52 C f$. la remise en cause de la domination de l'anglais dans le discours sur la traduction de M. Snell-Hornby. La question qu'elle a formulée dans son titre « Is Translation Studies going AngloSaxon?» (op. cit.), quoique posée dans un contexte différent, conduit à la même conclusion ; par ex., de nombreux périodiques scientifiques humanistes russes se tournent vers la publication (parfois exclusive) de textes en anglais.

53 Cela n'est probablement pas sans rapport avec la liquidation des maisons d'édition nationales à l'époque des bouleversements politiques. Concernant le contexte de la publication de traductions, voir : V. Komissarov, « Russian Tradition », p. 546. 
à un véritable échange d'idées. La thèse que les apports de la traductologie mondiale sont donc davantage importés en Russie par les commentaires auxquels ils donnent lieu (ce que j'ai essayé au moins de signaler) ou par la lecture des publications en langue originale que par le biais des traductions semble justifiée.

Dans la perspective d'un approfondissement de ces réflexions, la bibliométrie, l'analyse de citations et l'analyse qualitative des références aux ouvrages des chercheurs étrangers dans les textes russes pourraient être utiles ${ }^{54}$. Les différentes formes de transfert et leur impact éventuel sur le contenu des concepts " itinérants ${ }^{55}$ mériteraient également d'être étudiés.

Dans le cas des traditions traductologiques russe et occidentale, l'on ne peut pas parler de relation asymétrique. Ce sont deux puissants polysystèmes qui, durant des décennies, sont simplement restés presque entièrement étrangers l'un à l'autre. En Union soviétique, la science de la traduction était perçue comme un centre intégrant les idées de ses périphéries plurinationales. La dynamique de la réception des traductions de textes occidentaux de la discipline témoigne également d'une perception de soi-même en tant que centre, vu son caractère sélectif, imprévisible et aléatoire. Si elle se maintient, cette tendance isolationniste pourrait reléguer la traductologie russe dans la périphérie de la discipline.

Il est difficile de dire actuellement si le nombre accru de traductions de travaux occidentaux enregistrées au cours des dernières années est un signe de changement. Dans leurs considérations sur les faiblesses de la traductologie russe, Dmitrij Buzadži et Viktor Lančikov ne mentionnent pas d'incapacité ou de réticences à utiliser les concepts théoriques importés ${ }^{56}$. Dans leur article, ils signalent toutefois des utilisations non pertinentes de certaines méthodologies venues de l'étranger (cognitivisme, recherches de corpus), ce qui témoigne indirectement d'un échec du transfert de ces modèles de recherche à l'Est. Malgré l'explosion du nombre d'articles, de livres et de thèses de doctorat en traductologie, certains chercheurs russes éprouvent un sentiment de crise de la discipline. Une participation accrue des traductologues russes dans la circulation mondiale des idées pourrait peut-être les aider à le surmonter.

Traduit par Dorota Karczewska

54 Je m'attache partiellement à ces autres approches dans : M. Kaźmierczak, op. cit.

55 Voir L. D'hulst, op. cit., p. 138.

56 D. Buzadži, V. Lančikov, « Skorbnyj spisok. O bedah sovremennogo rossijskogo perevodovedeniâ », Mosty 4(36), 2012, pp. 42-56. 


\title{
A TRAVELLING THEORY? WESTERN TRANSLATION RESEARCH IN RUSSIAN TRANSLATION (PUBLISHING RECEPTION)
}

\begin{abstract}
The aim of this paper is to survey what texts and authors representing Western translation studies have been rendered into Russian over the last seven decades, and to describe the dynamics of the emergence of these translations as well as possible agendas behind the choices. The findings lead to the tentative conclusion that, especially in the $20^{\text {th }}$ century, translations were few (other means of translation studies knowledge transfer are touched upon). Renditions as such are only now beginning to play a part in the dissemination process.
\end{abstract}

Key words: Western translation studies, reception, circulation of scientific theories, publishing policies, Russia. 